\title{
Arthralgia among women taking aromatase inhibitors: is there a shared inflammatory mechanism with co-morbid fatigue and insomnia?
}

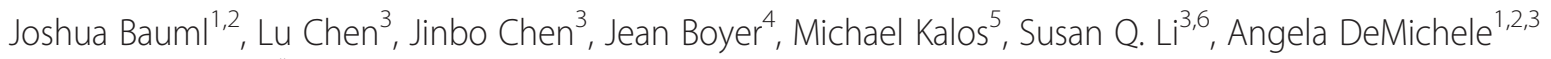
and Jun J. Mao ${ }^{1,3,6^{*}}$

\begin{abstract}
Introduction: Arthralgia is a common toxicity among women taking aromatase inhibitors (Als) and can lead to premature discontinuation of therapy. We evaluated the association between arthralgia, co-morbid fatigue and/or insomnia, and inflammatory biomarkers among women taking Als.

Methods: Women taking Als for early-stage breast cancer completed a modified version of the Brief Pain Inventory, the Brief Fatigue Inventory, and the Insomnia Severity Index and provided blood samples for simultaneous assessment of 34 inflammatory biomarkers with a Luminex kit. Two-sided $t$ tests were used to compare inflammatory biomarker concentrations for patients with or without moderate to severe arthralgia. Multivariate linear regression analyses were performed to evaluate the relationship between comorbid arthralgia, fatigue, and insomnia with identified biomarker concentrations.

Results: Among 203 participants, the severity of arthralgia, fatigue, and insomnia were significantly correlated with each other ( $p<0.001$ for all comparisons). After controlling for race, chemotherapy history, non-steroidal anti-inflammatory drug use, age, and body mass index, the coexistence of arthralgia, fatigue, and insomnia was associated with elevated $C$-reactive protein (CRP) $(\beta=93.1 ; 95 \%$ confidence interval $(C l): 25.1-161.1 ; p=0.008)$, eotaxin ( $\beta=79.9 ; 95 \% \mathrm{Cl}$ : 32.5-127.2; $p=0.001)$, monocyte chemoattractant protein (MCP)-1 ( $\beta=151.2 ; 95 \% \mathrm{Cl}$ : 32.7-269.8; $p=0.013$ ), and vitamin D-binding protein (VDBP) ( $\beta=19,422 ; 95 \% \mathrm{Cl}: 5500.5-33,344 ; p=0.006)$.

Conclusions: Among women taking Als, the coexistence of arthralgia, fatigue, and insomnia was associated with increased levels of inflammatory biomarkers (elevated CRP, eotaxin, MCP-1, and VDBP). These findings suggest a possible shared inflammatory mechanism underlying these common symptoms.
\end{abstract}

\section{Introduction}

Aromatase inhibitors (AIs) are an important component of the standard treatment of hormone receptor-positive breast cancer, but a high incidence of arthralgia (joint pain) may lead to premature therapy discontinuation and increased mortality [1,2]. Although up to $47 \%$ of women taking AIs report arthralgia [3], current understanding of the mechanisms underlying AI-associated arthralgia is limited.

\footnotetext{
* Correspondence: jun.mao@uphs.upenn.edu

'Abramson Cancer Center, University of Pennsylvania, Philadelphia, PA, USA

${ }^{3}$ Center for Clinical Epidemiology and Biostatistics and Department of Biostatistics and Epidemiology, Perelman School of Medicine, University of Pennsylvania, Philadelphia, PA, USA

Full list of author information is available at the end of the article
}

Menopause and other estrogen withdrawal syndromes are associated with an increase is systemic inflammation, which can exacerbate joint pain syndromes [4-8]. Given the precipitous drop in estrogen levels associated with AI administration [9], AI-associated arthralgia could be a result of similar immunomodulation. Imaging studies have revealed inflammatory tenosynovial changes at sites of AI-associated arthralgia, and a genome-wide association study revealed four single-nucleotide polymorphisms (SNPs) linking AI-associated arthralgia and T-cell maturation $[10,11]$. Prior efforts to evaluate the association between AI-associated arthralgia and systemic inflammation have been limited by small sample sizes [12, 13]. In contrast, fatigue and insomnia, which are often coexistent with pain in patients with cancer [14], have been 
associated with systemic inflammation [15-17]. In fact, the coexistence of arthralgia, fatigue, and insomnia is one of the more common symptom clusters experienced by breast cancer survivors [18, 19].

We aimed to evaluate the association of moderate to severe arthralgia with plasma inflammatory biomarker concentrations among postmenopausal women with early-stage breast cancer who were currently taking AIs. We hypothesized that the presence of moderate to severe arthralgia would be associated with elevated serum inflammatory biomarker concentrations. Because arthralgia often coexists with fatigue and insomnia [19], we then evaluated the association of the simultaneous experience of arthralgia, fatigue, and insomnia with inflammatory biomarkers. We hypothesized that the molecules identified in aim 1 would also be positively correlated with the coexistence of arthralgia, fatigue, and insomnia.

\section{Methods}

\section{Study design and patients}

We conducted a cross-sectional study, drawing our sample from an ongoing cohort study of patients receiving adjuvant AI therapy in the outpatient breast oncology clinic in the Abramson Cancer Center at the University of Pennsylvania between 2008 and 2013. Eligible participants were aged 18 years or older, had a diagnosis of early-stage breast cancer (stages I-III), were taking an AI, and had a Karnofsky score $\geq 60$ (i.e., ambulatory). Additional inclusion criteria required the completion of all chemotherapy and radiation therapy at least 1 month before enrollment, approval of the patient's treating oncologist, and the ability of the patient to understand and provide informed consent in English. After providing informed consent, patients completed a series of patient-reported outcome instruments and provided a non-fasting blood sample for analysis. Self-report questionnaires and chart abstraction were used for demographic and clinical information. For the present study, we included 203 participants who had had both survey data and a blood sample available during the time of biomarker analyses. The Institutional Review Board at the University of Pennsylvania approved this study.

\section{Symptom measurement}

Arthralgia was measured using a modified version of the Brief Pain Inventory (BPI). Because we were focused on arthralgia, the questions were modified to evaluate pain in and around joints. The BPI is validated in patients with cancer and is one of the most commonly used instruments used to measure pain. Domains measured are pain severity (four items) and interference (seven items), with Cronbach's $\alpha$ ranging from 0.8 to 0.87 and from 0.89 to 0.92 , respectively [20]. The use of a single item on the instrument, "Worst pain in the last 24 hours," has previously been validated for use of the BPI as a dichotomous measure to define moderate to severe pain [21]. Our group has previously shown that a cut point $\geq 4$ on this item was associated with greater likelihood to discontinue AI therapy prematurely [1].

Fatigue was measured using the Brief Fatigue Inventory (BFI). The BFI has been validated in cancer populations (Cronbach's $\alpha=0.96)$. Similar to the BPI, the "worst fatigue" item has been validated as a single-item dichotomous variable, with a cut point $\geq 4$ indicating moderate to severe fatigue [22]. Insomnia was measured using the Insomnia Severity Index, a seven-item survey validated in the assessment of insomnia severity among patients with cancer with a Cronbach's $\alpha$ ranging from 0.76 to 0.78 . A cut point $>14$ on the overall score has been validated as a dichotomous measure for the presence or absence of moderate to severe insomnia [23].

\section{Inflammatory biomarker measurement}

Serum levels of cytokines, chemokines, and inflammatory molecules were determined using the Cytokine Human Magnetic 30-Plex Panel for the Luminex platform (Life Technologies, Carlsbad, CA, USA) and the Acute Phase Human 4-Plex Panel for the Luminex platform (Life Technologies). With the 30-plex assay, we examined the following serum cytokine and chemokine levels: interleukin (IL)-1 $\beta$, IL-1RA, IL-2, IL-2R, IL-4, IL-5, IL-6, IL-7, IL-8, IL-10, IL-12p40/p70, IL-13, IL-15, IL-17, vascular endothelial growth factor, tumor necrosis factor $\alpha$, interferon (IFN) $-\alpha$, IFN- $\gamma$, granulocyte macrophage colony-stimulating factor, macrophage inflammatory protein (MIP)-1 $\alpha$, MIP1- $\beta$, IFN- $\gamma$-inducible protein 10 , monokine induced by interferon gamma, eotaxin, the regulated on activation, normal $\mathrm{T}$ expressed and secreted protein (RANTES), monocyte chemoattractant protein (MCP)-1, epidermal growth factor, granulocyte colonystimulating factor, basic fibroblast growth factor, and hepatocyte growth factor. Sera were defrosted on ice and added undiluted to the beads. Incubation and washing were carried out per assay specifications. With the 4-plex acute phase panel, we examined $\beta_{2}$-microglobulin, Creactive protein (CRP), haptoglobin, and vitamin $\mathrm{D}-$ binding protein (VDBP or Gc globulin). Sera were diluted as suggested to a 1:8000 concentration. The diluted sample was added to the magnetic beads, followed by incubation and washing per assay specifications.

\section{Statistical analysis}

Descriptive statistics were used to evaluate the distribution of scores on each of the three patient-reported outcome instruments, with which we measured arthralgia, fatigue, and insomnia. Patients were then dichotomized into the presence or absence of moderate to severe arthralgia, fatigue, and insomnia, as defined by the cut points 
described above. We performed $X^{2}$ analysis to assess pairwise correlations among the three symptoms.

We explored the variability in each inflammatory biomarker using box plots. We identified 20 biomarkers with limited intersubject variability. These were excluded from further analyses. We assessed pairwise correlations among the 14 remaining biomarkers, which revealed a significant association. To understand whether the variability in inflammatory biomarkers could be explained by clinical and demographic variables, we performed a series of linear regressions to evaluate the association of our collected clinical and demographic variables with inflammatory biomarker concentration. With our identified inflammatory biomarkers, we specifically assessed the association of each cytokine marker with age, race, non-steroidal anti-inflammatory drug (NSAID) use in the last 7 days, body mass index (BMI), education, years since last menstrual period, history of chemotherapy administration (with a specific focus on taxane history, given the risk for acute taxane induced neuropathy, which can present as joint pain), cancer stage, and years on AIs. Any variable with a $p$ value $\leq 0.1$ was included in our final model.

For the primary hypothesis, we conducted two-sided $t$ tests to compare the mean concentration of each inflammatory biomarker for patients with and without moderate to severe arthralgia. The Bonferroni adjustment was applied to adjust for multiplicity in testing $(p<0.004)$. For the secondary hypothesis, we created a composite binary variable to identify patients with all three symptoms versus those with fewer symptoms. We then conducted two-sided $t$ tests to compare the mean concentration of each inflammatory biomarker for patients with and without comorbid symptoms. Finally, we performed multivariate linear regression analysis to reevaluate the associations between symptoms and inflammatory biomarkers after adjusting for confounding variables.

\section{Results}

\section{Patient characteristics}

The demographic and clinical characteristics of study participants are shown in Table 1. Our analysis included 203 patients. Their mean age was 60.5 years, and $80.7 \%$ of them were white. The stage distribution was as follows: $47 \%$ in stage $0 / \mathrm{I}, 40.5 \%$ in stage II, and $12.5 \%$ in stage III. The most common AI used was anastrozole, which was used by $74.4 \%$ of participants. Chemotherapy had been administered to $61.7 \%$ of patients surveyed.

\section{Prevalence and correlation of key symptoms}

Using the cut points of moderate to severe symptoms defined above, $21.3 \%$ of patients had arthralgia, $41.6 \%$ of patients had fatigue, and $33.2 \%$ had insomnia. Arthralgia was significantly correlated with fatigue $(r=0.56, p<0.001)$, and $88.4 \%$ of patients with arthralgia also had fatigue. Arthralgia was also significantly correlated with insomnia $(r=0.49, p<0.001)$, and $83.7 \%$ of patients with arthralgia also had insomnia. Of the overall population, $16.8 \%$ of patients had all three symptoms. The demographic and clinical characteristics of study participants, as a function of the presence or absence of all three symptoms, are shown in Table 1.

\section{Association between arthralgia, fatigue, and insomnia with biomarkers}

The biomarkers exhibited significant pairwise correlation (Additional file 1: Table S1). As such, a multivariable model including all inflammatory biomarkers was not constructed. As can be seen in Figs. 1, 2, 3, and 4, higher concentrations of CRP $(p=0.0026)$, eotaxin $(p=0.0002)$, MCP-1 $(p=0.0017)$, and VDBP $(p=0.0006)$ were associated with moderate to severe arthralgia. These results were all statistically significant after correction for multiple comparisons. In an exploratory analysis of fatigue and insomnia, all of these biomarkers also showed an association with fatigue and insomnia, with the exception of a trend toward significance for CRP and insomnia. Additional file 2: Tables S2-S4 describe the results of all two-sided $t$ tests done to evaluate the association of each symptom with inflammatory biomarkers.

\section{Association of composite symptom variable with inflammatory biomarkers}

We performed a series of linear regressions to evaluate the association of our composite symptom burden variable with biomarker concentrations, controlling for BMI, race, chemotherapy status, NSAID use, and age. BMI, NSAID use, and race were associated with inflammatory biomarker concentrations in our sample (data not shown), whereas chemotherapy and age have been shown in other series to impact arthralgia and inflammatory biomarker concentrations $[15,24,25]$. CRP $(\beta=$ 93.1; $95 \%$ confidence interval [CI]: 25.1-161.1; $p=$ $0.008)$, eotaxin ( $\beta=79.9 ; 95 \%$ CI: 32.5-127.2; $p=0.001)$, MCP-1 $(\beta=151.2 ; 95 \%$ CI: 32.7-269.8; $p=0.013)$, and VDBP $(\beta=19,422 ; 95 \%$ CI: $5500.5-33,344 ; p=0.006)$ were strongly associated with the presence of all three symptoms in multivariate analyses (see Table 2).

\section{Discussion}

Arthralgia is a common side effect among women taking AIs and is associated with premature discontinuation of therapy [1]. Non-adherence to a 5-year regimen of AIs is associated with increased mortality [2]. Unfortunately, current understanding of this toxicity is limited. We found that moderate to severe arthralgia is associated with specific serum markers of inflammation (elevated CRP, eotaxin, MCP-1, and VDBP) among women taking 
Table 1 Participant characteristics $(N=203)$

\begin{tabular}{|c|c|c|c|}
\hline & Overall population & With symptom cluster & Without symptom cluster \\
\hline Mean age (SD) & $60.5(8.5) \mathrm{yr}$ & $60.1(9.4) \mathrm{yr}$ & $60.6(8.3) \mathrm{yr}$ \\
\hline \multicolumn{4}{|l|}{ Body mass index $\left(\mathrm{kg} / \mathrm{m}^{2}\right)$} \\
\hline Normal weight (18.5-24.9) & 83 (41.1\%) & $9(26.5 \%)$ & 74 (44 \%) \\
\hline Overweight (25-29.9) & $59(29.2 \%)$ & $12(35.3 \%)$ & $47(28 \%)$ \\
\hline Obese $(>30)$ & $60(29.7 \%)$ & $13(38.2 \%)$ & $47(28 \%)$ \\
\hline \multicolumn{4}{|l|}{ Race } \\
\hline White & $163(80.7 \%)$ & 26 (76.5\%) & 137 (81.5 \%) \\
\hline Non-white & $39(19.3 \%)$ & $8(23.5 \%)$ & 31 (18.5 \%) \\
\hline \multicolumn{4}{|l|}{ Education } \\
\hline High school or less & $46(22.8 \%)$ & $8(23.5 \%)$ & $38(22.6 \%)$ \\
\hline College & $93(46 \%)$ & 20 (58.8 \%) & $73(43.5 \%)$ \\
\hline Graduate/professional & $63(31.2 \%)$ & $6(17.7 \%)$ & 57 (33.9\%) \\
\hline \multicolumn{4}{|l|}{ Years since LMP } \\
\hline$<5 \mathrm{yr}$ & 41 (21.5\%) & $13(40.6 \%)$ & $80(50.3 \%)$ \\
\hline $5-10 \mathrm{yr}$ & $57(29.8 \%)$ & 10 (31.3\%) & 47 (29.6 \%) \\
\hline$>10 \mathrm{yr}$ & $93(48.7 \%)$ & $9(28.1 \%)$ & 32 (20.1\%) \\
\hline \multicolumn{4}{|l|}{ Cancer stage } \\
\hline Stage 0/l & $94(47 \%)$ & $13(38.2 \%)$ & $81(48.8 \%)$ \\
\hline Stage \| & $81(40.5 \%)$ & 16 (47.1\%) & 65 (39.2 \%) \\
\hline Stage III & $25(12.5 \%)$ & $5(14.7 \%)$ & $20(12 \%)$ \\
\hline \multicolumn{4}{|l|}{ Al taken } \\
\hline Anastrozole & $148(74.4 \%)$ & 27 (79.4 \%) & $121(73.3 \%)$ \\
\hline Exemestane & $20(10 \%)$ & 2 (5.9\%) & 18 (10.9\%) \\
\hline Letrozole & $31(15.6 \%)$ & $5(14.7 \%)$ & $26(15.8 \%)$ \\
\hline \multicolumn{4}{|l|}{ Years on Al } \\
\hline$<1 \mathrm{yr}$ & $54(26.7 \%)$ & $11(32.4 \%)$ & $43(25.6 \%)$ \\
\hline $1-3 \mathrm{yr}$ & 85 (42.1\%) & 12 (35.3 \%) & $73(43.5 \%)$ \\
\hline$>3 \mathrm{yr}$ & $63(31.2 \%)$ & 11 (32.3\%) & 52 (30.9 \%) \\
\hline \multicolumn{4}{|l|}{ Chemotherapy history } \\
\hline None & 77 (38.3 \%) & 14 (41.2 \%) & $63(37.7 \%)$ \\
\hline Regimen with a taxane & 78 (38.8 \%) & 10 (29.4\%) & 36 (21.6 \%) \\
\hline Regimen without a taxane & 46 (22.9\%) & 10 (29.4 \%) & 68 (40.7\%) \\
\hline \multicolumn{4}{|l|}{ NSAID use in last 7 days } \\
\hline Yes & $61(30.3 \%)$ & $17(50 \%)$ & 44 (26.3 \%) \\
\hline No & $138(68.7 \%)$ & $17(50 \%)$ & $121(72.5 \%)$ \\
\hline Don't know & $2(1 \%)$ & $0(0 \%)$ & $2(1.2 \%)$ \\
\hline
\end{tabular}

Al aromatase inhibitor, LMP last menstrual period, NSAID non-steroidal anti-inflammatory drug, SD standard deviation Symptom cluster refers to comorbid arthralgia, fatigue, and insomnia

AIs. We also found that the simultaneous experience of arthralgia, fatigue, and insomnia was associated with elevated serum biomarker concentrations. These data suggest that inflammation may be a shared mechanism of these toxicities.

Our findings are consistent with prior research tying estrogen deprivation to inflammation. Estrogen $\alpha$ - and $\beta$-receptors in the nucleus alter gene expression of various inflammatory biomarkers, leading to immunomodulation [5]. Indeed, estrogen supplementation seems to ameliorate certain autoimmune conditions [4], and women going through menopause can experience an exacerbation of inflammatory conditions, such as fibromyalgia and rheumatoid arthritis [7, 8]. 


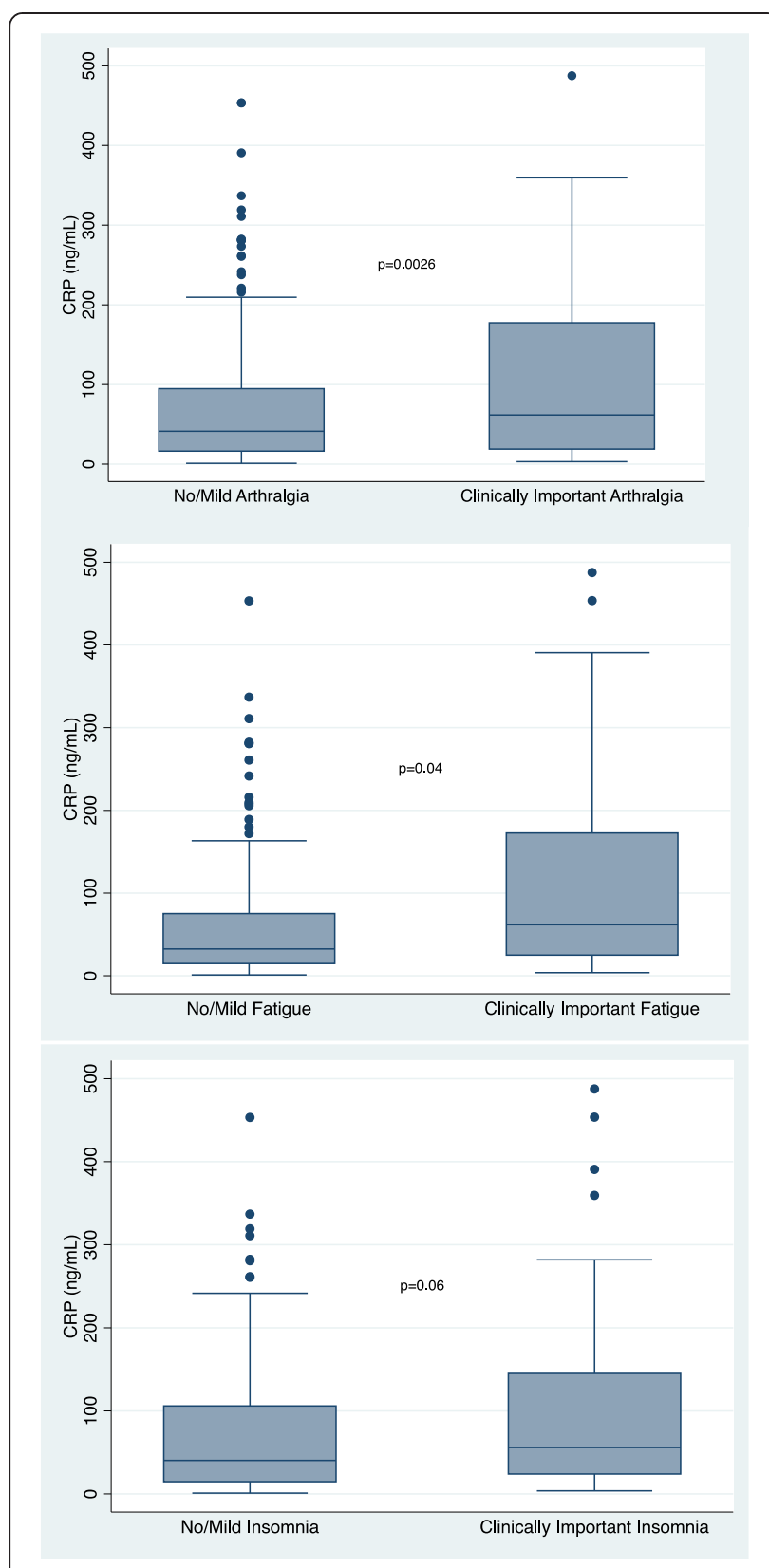

Fig. 1 Association of individual symptoms with C-reactive protein (CRP)

On the basis of this biological plausibility, others have evaluated the contribution of inflammation to AI-associated arthralgia. In a genome-wide association study, Ingle et al. identified a series of SNPs that were correlated with the presence of AI-associated arthralgia [11]. These SNPs centered on T-cell leukemia/ lymphoma protein 1A (TCL1A), an inflammatory protein whose activity is modulated by estrogen levels. The SNP variations associated with AI-associated arthralgia resulted in TCL1A levels that were more sensitive to estrogen variation. Researchers in two prior studies have explored the association of serum inflammatory biomarkers with

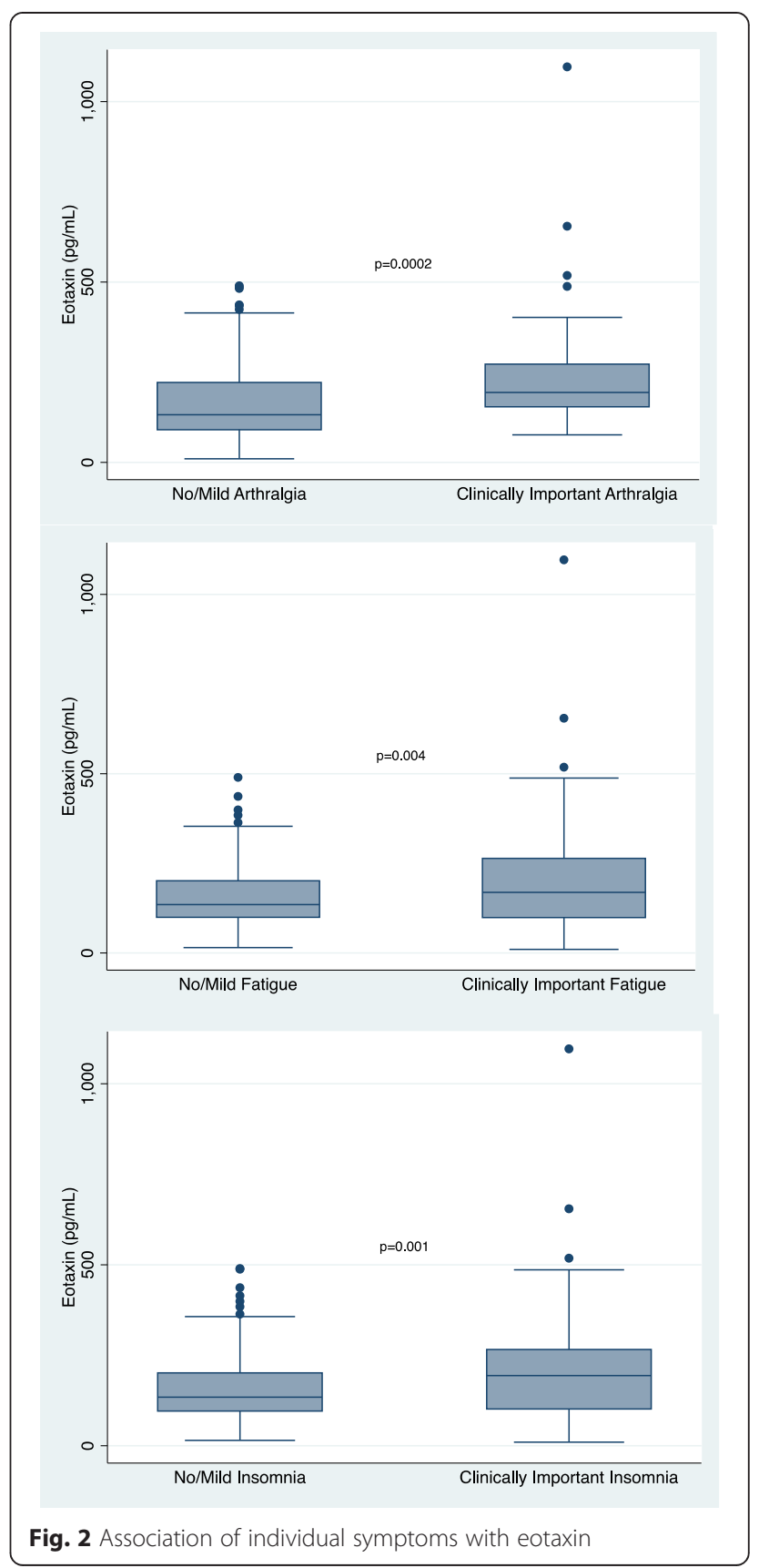

AI-associated arthralgia [12, 13]. Although neither of these studies revealed an association between arthralgia and systemic inflammation, both were limited by small sample sizes.

Our research found that arthralgia is significantly associated with fatigue and insomnia. Among those experiencing moderate to severe arthralgia, $88.4 \%$ also had fatigue and $83.7 \%$ also had insomnia. Bower et al. have evaluated the association of inflammatory biomarkers with fatigue both during [26] and after primary treatment [15] for breast cancer. Consistent with our findings, they noted that CRP elevations were associated with fatigue 


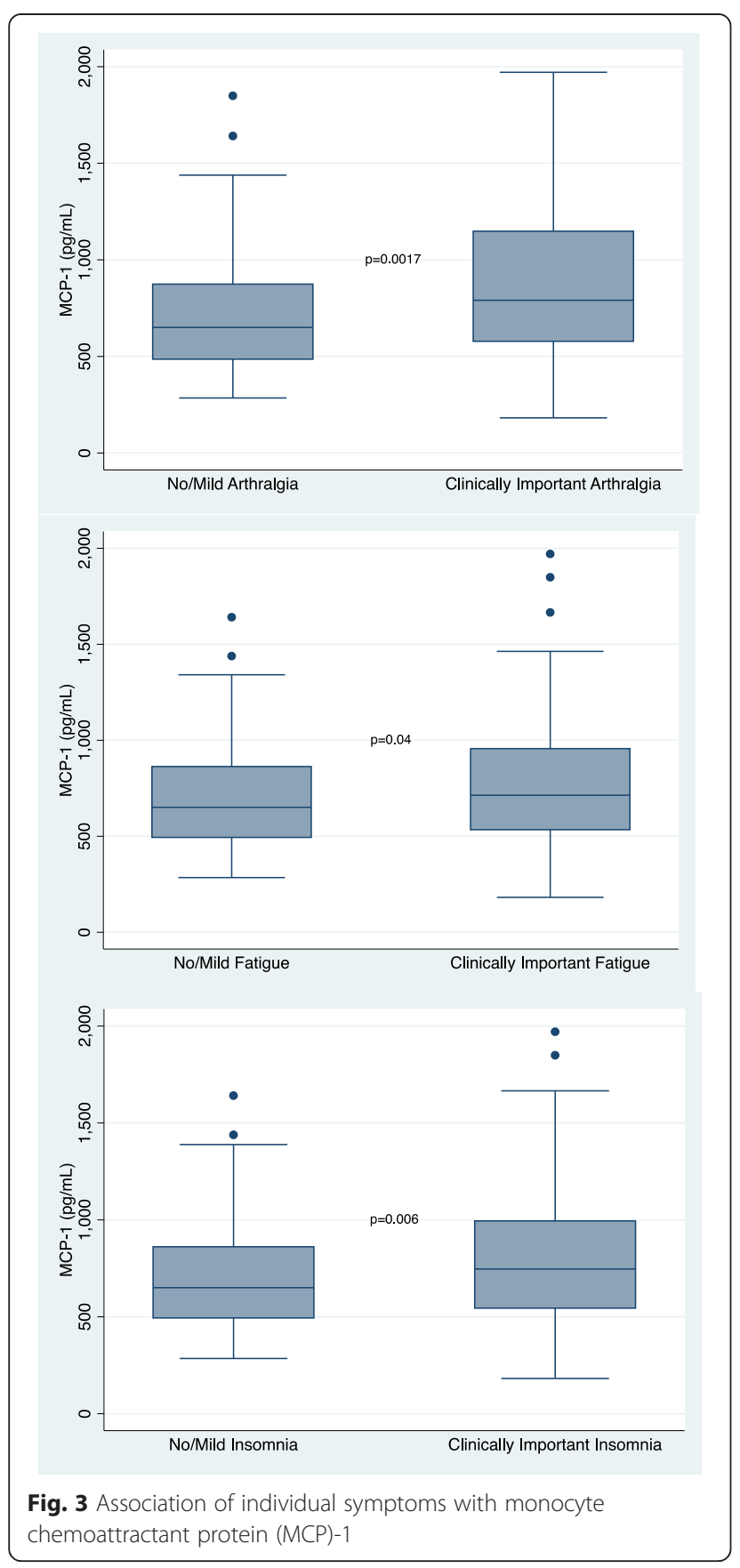

[26]. On a molecular level, estrogen blocks the expression of IL-6, which is the principal stimulatory molecule for the secretion of CRP $[5,6]$. It thus may not be surprising that patients have elevated CRP levels during treatment for breast cancer, when estrogen levels fall significantly. Our data suggest that these symptoms often coexist and that inflammation may underlie the mechanism of these common symptoms; therefore, interventions targeting inflammation may play a role in addressing these symptoms simultaneously.

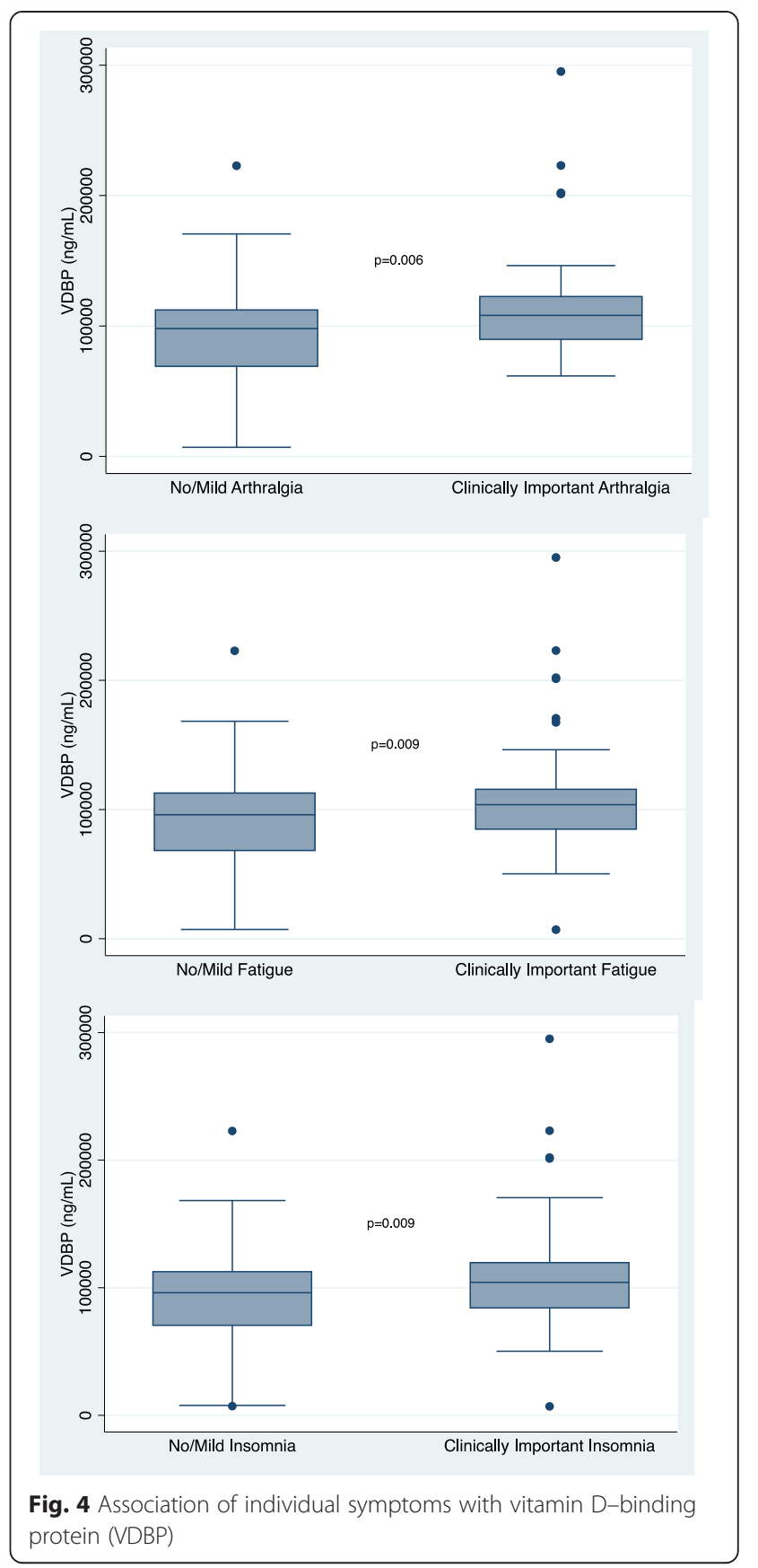

Our identification of VDBP, eotaxin, and MCP-1 as relevant inflammatory biomarkers for AI-related symptom burden is novel. VDBP is the primary binding protein for vitamin $\mathrm{D}$, but it is also an acute-phase reactant subject to significant genetic variability [27-31]. VDBP is thus a particularly intriguing mechanistic molecule, given accumulating evidence that vitamin $\mathrm{D}$ may have an important role in a wide variety of disease states [32]. Indeed, high-dose vitamin D supplementation was associated with a decreased incidence of $\mathrm{AI}$-associated arthralgia in one study [33]. Eotaxin and MCP-1 are chemokines 
Table 2 Association of symptom cluster (arthralgia, fatigue, and insomnia) with inflammatory biomarkers

\begin{tabular}{llllll}
\hline & \multicolumn{1}{l}{ Univariate analysis } & & & Multivariate analysis \\
\cline { 2 - 3 } & Coefficient $(95 \% \mathrm{Cl})$ & $p$ value & & Coefficient $(95 \% \mathrm{Cl})$ & 0.001 \\
\hline CRP & $117.3(49.3-185.3)$ & $<0.001$ & $79.9(32.5-127.2)$ & 0.008 \\
Eotaxin & $87.3(40.8-133.7)$ & 0.003 & $151.2(32.7-269.8)$ & 0.001 \\
MCP-1 & $178(63-293.2)$ & 0.002 & $19,422(5500.5-33,344)$ & 0.013 \\
VDBP & $21,530.8(8088.4-34,973.2)$ & 0.006 & \\
\hline
\end{tabular}

Data shown are results of multivariate linear regression analyses controlled for race, body mass index, chemotherapy status, non-steroidal anti-inflammatory drug use, and age

Cl confidence interval, CRP C-reactive protein, MCP-1 monocyte chemoattractant protein 1, VDBP vitamin D-binding protein

responsible for the recruitment of inflammatory cells to sites of injury $[34,35]$. Elevated eotaxin and MCP-1 concentrations have been seen in patients with fibromyalgia [36], another condition characterized by joint pain, insomnia, and fatigue [37].

Our study has a number of important limitations. First, it was cross-sectional in nature. As such, we are unable to establish the causal relationship between symptom burden and inflammatory biomarkers. Further prospective work is needed to clarify whether systemic inflammation leads to symptoms or if the persistent symptoms cause inflammation. Second, we examined numerous biomarkers in this study, and there is a risk of false-positive discovery. We adjusted our primary analysis using Bonferroni corrections for those biomarkers that demonstrated variability in our sample. A future replication study is needed to verify the results identified in our study. Third, our biomarker sampling was nonfasting, and we did not have complete data on comorbid conditions or other medications patients may have used, including statins and bisphosphonates. As described above, concurrent autoimmune conditions may also affect biomarker concentrations [36]. Future studies should control for these variables.

In spite of these limitations, this study is, to the best of our knowledge, the largest study to date evaluating the association of patient-reported symptoms with inflammatory biomarkers. We demonstrated that patientreported arthralgia and co-morbid fatigue and insomnia are associated with elevated serum inflammatory biomarkers among women taking AIs. A better understanding of this novel finding will allow clinicians to develop strategies to manage these symptoms to improve quality of life, adherence to AI therapies, and potentially survival outcomes for women with breast cancer.

\section{Conclusions}

Patient-reported arthralgia and co-morbid fatigue and insomnia were associated with elevated CRP, eotaxin, MCP-1, and VDBP among a group of breast cancer survivors taking AIs. A better understanding of symptomatic causes of distress will allow clinicians to develop strategies to manage these symptoms to improve quality of life, adherence to therapy, and potentially survival outcomes for women with breast cancer.

\section{Additional files}

Additional file 1: Table S1. Pairwise correlation between inflammatory biomarkers.

Additional file 2: Tables S2-S4. Two-sided $t$ tests of inflammatory biomarker concentrations as a function of symptoms.

\begin{abstract}
Abbreviations
Al: Aromatase inhibitor; BFI: Brief Fatigue Inventory; BMI: Body mass index; BPI: Brief Pain Inventory; Cl: Confidence interval; CRP: C-reactive protein; IFN: Interferon; IL: Interleukin; LMP: Last menstrual period; MCP-1: Monocyte chemoattractant protein 1; MIG: Monokine induced by interferon gamma; MIP: Macrophage inflammatory protein; NSAID: Non-steroidal antiinflammatory drug; RANTES: Regulated on activation, normal T expressed and secreted protein; SD: Standard deviation; SNP: Single-nucleotide polymorphism; TCL1A: T-cell leukemia/lymphoma protein 1A; VDBP: Vitamin D-binding protein; VEGF: Vascular endothelial growth factor.
\end{abstract}

\section{Competing interests}

The authors declare that they have no competing interests.

\section{Authors' contributions}

JBa participated in the design of the study, statistical analysis, and the drafting of the manuscript. LC and JC participated in the statistical analysis and the drafting of the manuscript. JBo performed the immunoassays and participated in the drafting of the manuscript. MK and AD participated in the design of the study and the drafting of the manuscript. SL participated in the study design, statistical analysis, and the drafting of the manuscript. JM participated in the design of the study, statistical analysis, and the drafting of the manuscript. All authors read and approved the final the manuscript.

\section{Acknowledgments}

This research is supported by grant R01 CA158243-01 from the National Cancer Institute, National Institutes of Health (to JJM, Principal Investigator).

\section{Author details}

${ }^{1}$ Abramson Cancer Center, University of Pennsylvania, Philadelphia, PA, USA. ${ }^{2}$ Department of Medicine, Division of Hematology/Oncology, Perelman School of Medicine, University of Pennsylvania, Philadelphia, PA, USA. ${ }^{3}$ Center for Clinical Epidemiology and Biostatistics and Department of Biostatistics and Epidemiology, Perelman School of Medicine, University of Pennsylvania, Philadelphia, PA, USA. ${ }^{4}$ Department of Pathology and Laboratory Medicine, Perelman School of Medicine, University of Pennsylvania, Philadelphia, PA, USA. ${ }^{5}$ Eli Lilly \& Company, New Hyde Park, NY, USA. 'Department of Family Medicine and Community Health, Perelman School of Medicine, University of Pennsylvania, 3400 Spruce Street, 2 Gates, Philadelphia, PA 19104, USA.

Received: 23 March 2015 Accepted: 15 June 2015

Published online: 28 June 2015 


\section{References}

1. Chim K, Xie SX, Stricker CT, Li QS, Gross R, Farrar JT, et al. Joint pain severity predicts premature discontinuation of aromatase inhibitors in breast cancer survivors. BMC Cancer. 2013;13:401.

2. Hershman DL, Shao T, Kushi LH, Buono D, Tsai WY, Fehrenbacher L, et al. Early discontinuation and non-adherence to adjuvant hormonal therapy are associated with increased mortality in women with breast cancer. Breast Cancer Res Treat. 2011;126:529-37.

3. Goss PE, Ingle JN, Martino S, Robert NJ, Muss HB, Piccart M, et al. A randomized trial of letrozole in postmenopausal women after five years of tamoxifen therapy for early-stage breast cancer. N Engl J Med. 2003;349:1793-802. doi:10.1056/NEJMoa032312. PubMed.

4. Carlsten $\mathrm{H}$. Immune responses and bone loss: the estrogen connection. Immunol Rev. 2005;208:194-206.

5. Straub $\mathrm{RH}$. The complex role of estrogens in inflammation. Endocr Rev. 2007;28:521-74.

6. Pfeilschifter J, Köditz R, Pfohl M, Schatz H. Changes in proinflammatory cytokine activity after menopause. Endocr Rev. 2002;23:90-119.

7. Goemaere S, Ackerman C, Goethals K, De Keyser F, Van der Straeten C, Verbruggen $\mathrm{G}$, et al. Onset of symptoms of rheumatoid arthritis in relation to age, sex and menopausal transition. J Rheumatol. 1990;17:1620-2.

8. Pamuk ON, Cakir N. The variation in chronic widespread pain and other symptoms in fibromyalgia patients: the effects of menses and menopause. Clin Exp Rheumatol. 2005;23:778-82.

9. Campos SM. Aromatase inhibitors for breast cancer in postmenopausal women. Oncologist. 2004:9:126-36.

10. Morales L, Pans S, Verschueren K, Van Calster B, Paridaens R, Westhovens R, et al. Prospective study to assess short-term intra-articular and tenosynovial changes in the aromatase inhibitor-associated arthralgia syndrome. J Clin Oncol. 2008;26:3147-52.

11. Ingle JN, Schaid DJ, Goss PE, Liu M, Mushiroda T, Chapman JA, et al. Genome-wide associations and functional genomic studies of musculoskeletal adverse events in women receiving aromatase inhibitors. J Clin Oncol. 2010;28:4674-82.

12. Henry NL, Pchejetski D, A'Hern R, Nguyen AT, Charles P, Waxman J, et al. Inflammatory cytokines and aromatase inhibitor-associated musculoskeletal syndrome: a case-control study. Br J Cancer. 2010;103:291-6.

13. Laroche F, Coste J, Medkour T, Cottu PH, Pierga JY, Lotz JP, et al. Classification of and risk factors for estrogen deprivation pain syndromes related to aromatase inhibitor treatments in women with breast cancer: a prospective multicenter cohort study. J Pain. 2014;15:293-303.

14. Miaskowski C, Cooper BA, Paul SM, Dodd M, Lee K, Aouizerat BE, et al. Subgroups of patients with cancer with different symptom experiences and quality-of-life outcomes: a cluster analysis. Oncol Nurs Forum. 2006:33:E79-89.

15. Bower JE, Ganz PA, Irwin MR, Kwan L, Breen EC, Cole SW. Inflammation and behavioral symptoms after breast cancer treatment: do fatigue, depression, and sleep disturbance share a common underlying mechanism? J Clin Oncol. 2011;29:3517-22.

16. Bower JE, Ganz PA, Irwin MR, Castellon S, Arevalo J, Cole SW. Cytokine genetic variations and fatigue among patients with breast cancer. J Clin Oncol. 2013;31:1656-61.

17. Rogers LQ, Fogleman A, Trammell R, Hopkins-Price P, Spenner A, Vicari S, et al. Inflammation and psychosocial factors mediate exercise effects on sleep quality in breast cancer survivors: pilot randomized controlled trial. Psychooncology. 2014;24:302-10.

18. Pachman DR, Barton DL, Swetz KM, Loprinzi CL. Troublesome symptoms in cancer survivors: fatigue, insomnia, neuropathy, and pain. J Clin Oncol. 2012;30:3690-6.

19. van Nes JG, Fontein DB, Hille ET, Voskuil DW, van Leeuwen FE, de Haes JC, et al. Quality of life in relation to tamoxifen or exemestane treatment in postmenopausal breast cancer patients: a Tamoxifen Exemestane Adjuvant Multinational (TEAM) Trial side study. Breast Cancer Res Treat. 2012;134:267-76.

20. Cleeland CS, Ryan KM. The Brief Pain Inventory. Houston, TX: Pain Research Group, The University of Texas MD Anderson Cancer Center; 1991.

21. Atkinson TM, Mendoza TR, Sit L, Passik S, Scher HI, Cleeland C, et al. The Brief Pain Inventory and its "pain at its worst in the last 24 hours" item: clinical trial endpoint considerations. Pain Med. 2010;11:337-46.
22. Mendoza TR, Wang XS, Cleeland CS, Morrissey M, Johnson BA, Wendt JK, et al. The rapid assessment of fatigue severity in cancer patients: use of the Brief Fatigue Inventory. Cancer. 1999;85:1186-96.

23. Savard MH, Savard J, Simard S, Ivers H. Empirical validation of the Insomnia Severity Index in cancer patients. Psychooncology. 2005;14:429-41.

24. Forsey RJ, Thompson JM, Ernerudh J, Hurst TL, Strindhall J, Johansson B, et al. Plasma cytokine profiles in elderly humans. Mech Ageing Dev. 2003;124:487-93.

25. Mao JJ, Stricker C, Bruner D, Xie S, Bowman MA, Farrar JT, et al. Patterns and risk factors associated with aromatase inhibitor-related arthralgia among breast cancer survivors. Cancer. 2009;115:3631-9.

26. Bower JE, Ganz PA, Tao ML, Hu W, Belin TR, Sepah S, et al. Inflammatory biomarkers and fatigue during radiation therapy for breast and prostate cancer. Clin Cancer Res. 2009;15:5534-40.

27. Powe $C E$, Evans MK, Wenger J, Zonderman AB, Berg AH, Nalls M, et al. Vitamin D-binding protein and vitamin D status of black Americans and white Americans. N Engl J Med. 2013:369:1991-2000.

28. Speeckaert M, Huang G, Delanghe JR, Taes YE. Biological and clinical aspects of the vitamin D binding protein (Gc-globulin) and its polymorphism. Clin Chim Acta. 2006;372:33-42.

29. Meier U, Gressner O, Lammert F, Gressner AM. Gc-globulin: roles in response to injury. Clin Chem. 2006;52:1247-53.

30. Malik S, Fu L, Juras DJ, Karmali M, Wong BYL, Gozdzik A, et al. Common variants of the vitamin $D$ binding protein gene and adverse health outcomes. Crit Rev Clin Lab Sci. 2013;50:1-22.

31. Abbas S, Linseisen J, Slanger T, Kropp S, Mutschelknauss EJ, Flesch-Janys D, et al. The Gc2 allele of the vitamin D binding protein is associated with a decreased postmenopausal breast cancer risk, independent of the vitamin D status. Cancer Epidemiol Biomarkers Prev. 2008;17:1339-43.

32. Holick MF. Vitamin D, deficiency. N Engl J Med. 2007;357:266-81.

33. Khan QJ, Reddy PS, Kimler BF, Sharma P, Baxa SE, O'Dea AP, et al. Effect of vitamin D supplementation on serum 25-hydroxy vitamin D levels, joint pain, and fatigue in women starting adjuvant letrozole treatment for breast cancer. Breast Cancer Res Treat. 2010;119:111-8.

34. Bradding P, Walls AF, Holgate ST. The role of the mast cell in the pathophysiology of asthma. J Allergy Clin Immunol. 2006;117:1277-84.

35. Carr MW, Roth SJ, Luther E, Rose SS, Springer TA. Monocyte chemoattractant protein 1 acts as a T-lymphocyte chemoattractant. Proc Natl Acad Sci U S A. 1994:91:3652-6.

36. Zhang Z, Cherryholmes G, Mao A, Marek C, Longmate J, Kalos M, et al. High plasma levels of MCP-1 and eotaxin provide evidence for an immunological basis of fibromyalgia. Exp Biol Med. 2008;233:1171-80.

37. Nicassio PM, Moxham EG, Schuman CE, Gevirtz RN. The contribution of pain, reported sleep quality, and depressive symptoms to fatigue in fibromyalgia. Pain. 2002;100:271-9.

\section{Submit your next manuscript to BioMed Central and take full advantage of:}

- Convenient online submission

- Thorough peer review

- No space constraints or color figure charges

- Immediate publication on acceptance

- Inclusion in PubMed, CAS, Scopus and Google Scholar

- Research which is freely available for redistribution 